\title{
Incorporation of Benzothiadiazole Moiety at Junction of Polyfluorene- Polytriarylamime Block Copolymer for Effective Color Tuning in Organic Light Emitting Diode
}

Kyusun Kim, Yohei Inagaki, Shinji Kanehashi*, and Kenji Ogino*

Graduate School of Bio-Applications and Systems Engineering, Tokyo University of Agriculture and Technology, 2-24-16 Nakacho, Koganei-shi, Tokyo 184-8588, Japan

E-mail:kanehasi@cc.tuat.ac.jp, kogino@cc.tuat.ac.jp

\section{ABSTRACT}

Block copolymer consisting of polyfluorene and polytriarylamine with benzothiadiazole moiety at the junction is prepared in order to obtain an orange emitting polymer via Suzuki-

Miyaura followed by Buchwald-Hartwig coupling reactions. Electroluminescent device fabricated with resulting block copolymer exhibit only orange emission, although slight blue emission is observed in the fluorescent spectrum for the thin film, indicating that benzothiadiazole part plays a role of an effective trap site. Devices based on polyfluorene homopolymer doped with block copolymer $(10 \mathrm{wt} \%)$ or an orange emitting model compound at the corresponding content of benzotiadiazole unit are also fabricated. The device with the model compound exhibits orange emission with CIE coordinate of $(0.58,0.42)$, whereas that with block copolymer pale orange with the coordinate of $(0.44,0.38)$. This fact is probably due to the preferential distribution of block copolymer at the vicinity of anode via hydrophilic interaction of trioxyethylene side chains with poly $(3,4-$ ethylenedioxythiophene):poly(styrenesulfonate) (PEDOT:PSS).

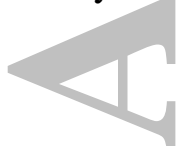

Keywords: electroluminescence; block copolymer; junction; benzothiadiazole; polymer lightemitting diode (PLED)

This is the author manuscript accepted for publication and has undergone full peer review but has not been through the copyediting, typesetting, pagination and proofreading process, which may lead to differences between this version and the Version record. Please cite this article as doi:10.1002/ app.45393. 


\section{Introduction}

Polymer light-emitting diodes (PLEDs) have attracted much attention from the scientific and technological view points since their first discovery in 1990 [1]. Compared with conventional low molecular mass materials in electroliminescent (EL) applications, there are several advantages in polymeric materials, i.e., inexpensive wet-processes such as spin-coating and ink-jet can be applied [2]. Since EL performance in PLED, however, is generally poorer compared with devices based on low molecular mass materials, innovative molecular designs for polymeric materials are indispensable for the practical applications. Polyfluorene (PF)

based materials have been investigated as blue light emitting materials in PLEDs because of their superior properties, such as highly efficient photoluminescence (PL), excellent thermal stability, and good solubility in common organic solvents [3]. In order to overcome a serious problem assosiated with PF due to an imbalance in charge carriers caused by large hole injection barriers and different charge carrier mobilities, hole transporting triarylamine units have been incorporated into the PF backbones [4].

As a novel molecular design, we proposed block copolymer (BC) strategies, and reported BCs consisting of PF and polytriarylamine (PTAA) with trioxyethylene substituents [5], which were synthesized via the combination of Suzuki-Miyaura and C-N coupling polymerizations [6]. Emission efficiencies in devices based on block copolymers increased due to the facile hole injection from the anode and/or the efficient electron block by PTAA moieties. It is strongly expexted that PTAA block locates in the vicinity of the poly $(3,4-$ ethylenedioxythiophene):poly(styrenesulfonate) (PEDOT:PSS) coated on the anode via an interfacial segregation through the hydrogen bonding of trioxyethylene group with PSS [7].

A number of studies have been reported about the tuning of the emission colors by introducing a variety of narrow bandgap moieties to the PF backbone [8-19] or side chain [20,21] or doping chromophores [22-24], and various types of polymers have been applied to 
white PLED in single component polymer [10-14, 21] or blend [15]. As narrow band-gap comonomers, 2,1,3-benzothiadiazole (BT) moiety has often been utilized [8-15, 20, 21]. In the most reported polymers, narrow band gap comonomers are reasonably considered to be incorporated in a random manner in the main- or side chains except our previous work [25].

When the content of comonomer is very low and molecular weight of synthesized polymer is not so high, there should be intermolecular heterogeneity statistically. The effect of chemical compositional distribution of a copolymer has been unexplored in this field.

In this study, PTAA- $b$-PF- $b$-PTAA block copolymer functionalized with BT moiety at the junction point of two blocks was synthesized in order to obtain an orange light emitting material with the well-defined structure, and optical properties and EL characteristics were investigated for the resulting polymer as well as blends with PF homopolymer. In the case of blend based devices, remarkable difference was observed between the device based on PF doped with the block copolymer and that with the low molecular dye, which can be explained by the segregation of hydrophilic PTAA near the anode covered with PEDOT/PSS.

\section{Experimental Section}

\subsection{Materials}

All reagents and solvents were used without further purification unless stated otherwise. Tetrahydrofuran (THF) was distilled over sodium and benzophenone, and stored under nitrogen atmosphere. Toluene was distilled over calcium hydride, and stored under nitrogen atmosphere. $o$-Dichlorobenzene was also distilled over calcium hydride, and stored under nitrogen atmosphere. 2,7-Dibromofluorene (1), 2,7-dibromofluoren-9-one (2), 2,7-dibromo-9(4-octylphenyl)fluoren-9-ol (3), 2,7-dibromo-9-(4-methylphenyl)-9-(4-octylphenyl)fluorene (4), 2,7-bis(4,4,5,5-tetramethyl-1,3,2-dioxaborolan-2-yl)-9-(4-methylphenyl)-9-(4octylphenyl)fluorene (5), 1-\{2-(2-(2-methoxyethoxy)ethoxy) ethoxy $\}-4-$ nitrobenzene (6), 4\{2-[2-(2-methoxyethoxy)ethoxy]ethoxy\}phenylamine (7), (4'-bromobiphenyl-4-yl)-(4-\{2-[2- 
(2-methoxyethoxy)ethoxy]ethoxy\}phenyl)amine (8) were prepared as described elsewhere [5]. 4,7-Dibromobenzo[1, 2, 5]thiadiazole (9), 4,7-diphenyl- benzo[1, 2, 5]thiadiazole (10), 4,7bis(4'-bromophenyl) benzo[1,2,5]thiadiazole (11), 4-(4'-bromophenyl)-7-[4' '-(4', 'butylphenylamino) phenyl]-2,1,3-benzothiadiazole (12), and 4,7-bis(4-(N-phenyl-N-(4butylphenyl)amino)phenyl)-2,1,3-benzothiadiazole (13) were prepared as previously reported [26]. Polyfluorene homopolymer (PF) was prepared via Suzuki-Miyaura coupling polymerization with monomers (4) and (5) [26].

\section{Synthesis of Block Copolymer (BP) Precursor}

To a $10-\mathrm{mL}$, round-bottom flask equipped with a stopcock and a condenser were added 4 (0.0787 g, $0.131 \mathrm{mmol}), 5$ (0.100 g, $0.143 \mathrm{mmol}), 12(0.010 \mathrm{~g}, 0.020 \mathrm{mmol}), \mathrm{Pd}\left(\mathrm{PPh}_{3}\right)_{4}$ $(0.001 \mathrm{~g}, 0.5 \mathrm{~mol} \%), 2 \mathrm{M} \mathrm{K}_{2} \mathrm{CO}_{3}$ aq. $(2 \mathrm{~mL})$ and toluene $(2 \mathrm{~mL})$ under nitrogen atmosphere, and the mixture was stirred and heated at $80^{\circ} \mathrm{C}$ for $72 \mathrm{~h}$. After the reaction, toluene was added, the resulting solution was washed with sat. $\mathrm{NaCl}$ aq. and dried with $\mathrm{MgSO}_{4}$. The

solvent was removed by rotary evaporator, BP precursor was obtained by precipitation into methanol, and washed with acetone utilizing Soxhlet extraction apparatus for $24 \mathrm{~h}$. The yield was $0.093 \mathrm{~g}(70.2 \%) .{ }^{1} \mathrm{H} \mathrm{NMR}\left(400 \mathrm{MHz}, \mathrm{CDCl}_{3}\right) \delta(\mathrm{ppm}): 8.06-7.89(d d, J=8.24 \mathrm{~Hz}, 4 \mathrm{H}$; Ar H), $7.84-7.69$ (m, 48H; Ar H), $7.65-7.43(m, 101 \mathrm{H} ; \mathrm{Ar} \mathrm{H}), 7.23-7.11(m, 86 \mathrm{H} ; \mathrm{Ar} \mathrm{H})$, 7.09 - $6.93(m, 76 \mathrm{H} ; \mathrm{Ar} \mathrm{H}), 5.83(s, 1 \mathrm{H} ; \mathrm{NH}), 2.63-2.46\left(\mathrm{~m}, 67 \mathrm{H} ; \mathrm{CH}_{2}\right), 2.3\left(s, 75 \mathrm{H} ; \mathrm{CH}_{3}\right)$, $1.68-1.5\left(m, 59 \mathrm{H} ; \mathrm{CH}_{2}\right), 1.47-1.16\left(m, 262 \mathrm{H} ; \mathrm{C}_{5} 5 \mathrm{H}_{10}\right), 0.95\left(t, J=7.3 \mathrm{~Hz}, 3 \mathrm{H} ; \mathrm{CH}_{3}\right), 0.9-$ $0.81\left(m, 75 \mathrm{H} ; \mathrm{CH}_{3}\right)$

\section{Synthesis of Block Copolymer (BP)}

To a 20-mL two-necked round-bottom flask equipped with a stopcock and a condenser were added BP precursor (0.078 g, $0.012 \mathrm{mmol}), 8$ (0.119 g, $0.30 \mathrm{mmol}), \mathrm{Pd}(\mathrm{OAc})_{2}(0.0014 \mathrm{~g}$, $0.006 \mathrm{mmol}), t$ - BuONa $(0.032 \mathrm{~g}, 0.33 \mathrm{mmol})$ and toluene $3 \mathrm{~mL}$ under nitrogen atmosphere, 
and freeze pump thawed for 3 times, then added $\mathrm{P}(t-\mathrm{Bu})_{3}(7.5 \mu \mathrm{L}, 0.01 \mathrm{mmol})$. The mixture was stirred under reflux for $24 \mathrm{~h}$. After removing the solvent by rotary evaporator, the product was obtained by precipitation into methanol and washed by acetone soxhlet for $24 \mathrm{~h}$. The yield of BP was $0.111 \mathrm{~g}(63.9 \%) .{ }^{1} \mathrm{H}$ NMR $\left(400 \mathrm{MHz}, \mathrm{CDCl}_{3}\right) \delta(\mathrm{ppm}): 8.1-7.8(\mathrm{~m}, 4 \mathrm{H} ; \mathrm{Ar}$ H), 7.79 - $7.64(m, 30 H ; A r H), 7.61-7.34(m, 173 \mathrm{H} ; \mathrm{Ar} \mathrm{H}), 7.22$ - $6.94(m, 324 \mathrm{H} ; \mathrm{Ar} \mathrm{H})$, 6.92 - 6.77 (m, 42H; Ar H), $4.13\left(m, 51 \mathrm{H} ; \mathrm{CH}_{2} \mathrm{O}\right), 3.86\left(m, 50 \mathrm{H} ; \mathrm{CH}_{2} \mathrm{O}\right), 3.75(m, 51 \mathrm{H}$; $\left.\mathrm{CH}_{2} \mathrm{O}\right), 3.67\left(m, 53 \mathrm{H} ; \mathrm{CH}_{2} \mathrm{O}\right), 3.56\left(m, 51 \mathrm{H} ; \mathrm{CH}_{2} \mathrm{O}\right), 3.38\left(s, 71 \mathrm{H}, \mathrm{CH}_{3}\right), 2.6-2.4(m, 56 \mathrm{H}$; $\left.\mathrm{CH}_{2}\right), 2.22\left(s, 75 \mathrm{H} ; \mathrm{CH}_{3}\right), 1.8-1.45\left(m, 84 \mathrm{H} ; \mathrm{CH}_{2}\right), 1.41-1.15\left(m, 260 \mathrm{H} ; \mathrm{C}_{5} \mathrm{H}_{10}\right), 0.95(m$, $\left.3 \mathrm{H} ; \mathrm{CH}_{3}\right), 0.9-0.8\left(m, 75 \mathrm{H} ; \mathrm{CH}_{3}\right)$.

\section{Syntheis of 4,7-bis(4-( $N$-phenyl- $N$-(4-butylphenyl)amino)phenyl)-2,1,3-}

\section{benzothiadiazole (13)}

To a 100-mL two-necked round-bottom flask equipped with a stopcock and a condenser were added 4,7-bis-(4-bromophenyl)-benzo[l, $2, \quad 5]$ thiadiazole $\quad(0.7 \quad \mathrm{~g}, \quad 1.57$ mmol), 4butyldiphenylamine $(1.5 \mathrm{~g}, 6.3 \mathrm{mmol}), \mathrm{Pd}(\mathrm{OAc})_{2}(0.007 \mathrm{~g}, 0.04 \mathrm{mmol}), t$-BuONa $(0.15 \mathrm{~g}, 1.7$ mmol) and toluene $30 \mathrm{~mL}$ under nitrogen atmosphere and freeze pump thawed for 3 times, then added $\mathrm{P}(t-\mathrm{Bu})_{3}(0.3 \mathrm{~mL}, 0.4 \mathrm{mmol})$. The mixture was stirred under reflux for $24 \mathrm{~h}$. After the reaction, the resulting solution was washed by toluene. After removing the solvent by rotary evaporator, the product was obtained by column chromatography (hexane : toluene = 1:1). The yield was $0.111 \mathrm{~g}(64 \%) .{ }^{1} \mathrm{H}$ NMR (300 $\left.\mathrm{MHz}, \mathrm{CDCl}_{3}\right) \delta(\mathrm{ppm}): 7.89(d, J=8.4 \mathrm{~Hz}$, 2H), $7.75(s, 1 \mathrm{H}), 7.25(m, 2 \mathrm{H}), 7.21(m, 2 \mathrm{H}), 7.08(m, 2 \mathrm{H}), 2.62(t, J=7.5 \mathrm{~Hz}, 2 \mathrm{H}), 1.65$ (six, $J=7.5 \mathrm{~Hz}, 2 \mathrm{H}), 1.42$ (six, $J=7.5 \mathrm{~Hz}, 2 \mathrm{H}), 0.94(t, J=7.5 \mathrm{~Hz}, 3 \mathrm{H})$.

\subsection{Characterization}

${ }^{1} \mathrm{H}-\mathrm{NMR}$ spectra were obtained on a JEOL ECX 300, 400 instrument at $300 \mathrm{MHz}, 400 \mathrm{MHz}$ at $25^{\circ} \mathrm{C}$. Deutrated chloroform was used as a solvent with tetramethylsilane as an internal 
standard. Number and weight-average molecular weights $\left(M_{\mathrm{n}}\right.$ and $\left.M_{\mathrm{w}}\right)$ and polydispersity index (PDI) were estimated by gel permeation chromatography (GPC) analysis with a JASCO RI-2031 detector eluted with chloroform at a flow rate of $0.5 \mathrm{~mL} / \mathrm{min}$ at room temperature and calibrated by standard polystyrene samples. Differential scanning calorimetry (DSC) analyses were performed on a Rigaku DSC-8230 under nitrogen atmosphere at heating and cooling rates of $10^{\circ} \mathrm{C} / \mathrm{min}$. UV-vis absorption spectra were obtained on a JASCO V-570

spectrophotometer, and photoluminescent (PL) spectra were obtained with a JASCO FP-6500 spectrophotometer with an excitation at $380 \mathrm{~nm}$. The concentration of solution was $1 \mathrm{mg} / \mathrm{L}$ in chloroform. The solid films of polymer samples were prepared on a glass substrate by a spin coating method from chlorobenzene solutions $(10 \mathrm{mg} / \mathrm{mL})$ at room temperature. We examined BP, and blend samples (Blend 1: 5\% BP in PF, Blend 2: $10 \%$ of BP in PF, and Blend 3: PF with 0.4 wt\% of compound 13). Cyclic voltammetry $(\mathrm{CV})$ measurements were conducted at room temperature on a Hokuto denko Model HZ-5000 potentiogalvanostat equipped with a typical three-electrode cell with a working electrode (glassy carbon electrode), a reference electrode $(\mathrm{Ag} / \mathrm{AgCl})$, and a counter electrode ( $\mathrm{Pt}$ wire) at a scanning rate of $0.1 \mathrm{~V} / \mathrm{s}$. The measumenre was performed using thin film prepared on a working electrode by dip-coating method. Highest occupied molecular orbital (HOMO) and lowest unoccupied molecular orbital (LUMO) level were determined from the onset of the oxidation and reduction peaks.

\subsection{Device Fabrication}

Four kinds of devices were fabricated with a structure of ITO/poly(3,4ethylenedioxythiophene):polystyrene sulfonate (PEDOT:PSS) $(30 \mathrm{~nm}) /$ active layer (100 $\mathrm{nm}) / \mathrm{BCP}(50 \mathrm{~nm}) / \mathrm{LiF}(0.5 \mathrm{~nm}) / \mathrm{Al}(100 \mathrm{~nm})$ configuration. ITO substrates were pre-cleaned in an ultrasonic bath of detergent, methanol, deionized water, 2-propanol and acetone for 15 min sequentially. PEDOT:PSS was spin-coated onto a patterned indium tin oxide (ITO) at the 
speed of $2500 \mathrm{rpm}$ for $60 \mathrm{~s}$, and annealed at $200{ }^{\circ} \mathrm{C}$ for $1 \mathrm{~h}$. The active layer was spin-coated on PEDOT:PSS coated substrate from the polymer solution in chlorobenzene at speeds of $1000 \mathrm{rpm}$ for $30 \mathrm{~s}$ respectively and annealed at $120{ }^{\circ} \mathrm{C}$ for $1 \mathrm{~h}$ under argon atmosphere. A 50 $\mathrm{nm}$ thick layer of bathocuproine (BCP) and a $0.5 \mathrm{~nm}$ thick layer of lithium fluoride (LiF) followed with a $100 \mathrm{~nm}$ thick top contact of aluminum (Al) were thermally evaporated onto the active layer in a vacuum chamber under $10^{-5} \mathrm{~Pa}$. The thickness of each layer was measured by Stylus profilometer (DektakXT). The typical active area of the EL devices was 4-8 $\mathrm{mm}^{2}$. PLED performance was evaluated using Keithley 2400 source measurement unit and LS-100 spectroradiometer (Konica Minolta).

\section{Results and Discussion}

\subsection{Synthesis and Characterization}

The polymerization conditions and characteristics of synthesized polymers are summarized in Table 1. Conventional blue emitting polyfluorene (PF) was synthesized by Suzuki-Miyaura cross-coupling polymerization as described elsewhere [5]. Block copolymer (BP) consisting of PF and PTAA modified with BT moiety at the junction points was synthesized shown in Scheme 1. First, PF with amino functionalized BT moiety at both chain ends was prepared via Suzuki-Miyaura cross-coupling using conventional monomers for PF synthesis in the presence of BT containing bromide with diphenyl amine moiety [26], where the stoichiometric balance between bromide and borate groups was kept. As shown in ${ }^{1} \mathrm{H}-\mathrm{NMR}$ for the precursor (Figure 1a), small signals at $0.95,5.80,7.90$, and $8.02 \mathrm{ppm}$ assigned to diphenylamine chain ends appeared confirming the introduction of BT moiety with diphenylamine unit. Assuming that produced polymer is terminated with diphenylamine moiety, number average degree of polymerization is estimated to be 50 by comparison of signal intensities for two methyl groups. Then, this precursor was converted to block copolymer via Buchwald-Hartwig reaction ( $\mathrm{C}-\mathrm{N}$ coupling) with $\mathrm{AB}$ type of brominated 
diarylamine monomer with hydrophilic trioxyethylene (TEO) substituent according to the literature.[5] As shown in Figure 1b, ${ }^{1} \mathrm{H}-\mathrm{NMR}$ of $\mathrm{BP}$ exhibited signals assignable to methylene protons derived from TEO chain observed at 3.3-4.2 ppm, clearly indicating that TAA monomer 4 was introduced to form BP as designed. The molar ratio of the repeating unit of BP estimated from ${ }^{1} \mathrm{H}-\mathrm{NMR}$ based on the area integral of ether proton and aliphatic proton was 52:48 (PF : PTAA). All the polymers were soluble in common organic solvents süch as chloroform and THF, indicating that solution processes can be applied for the thin film fabrication.

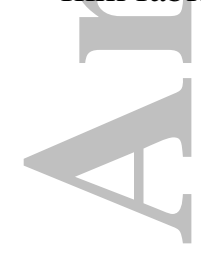

Table 1

Scheme 1

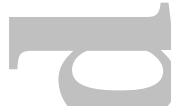

Figure 1

Figure 2 represents the GPC traces of BP and its precursor. Both polymers showed the mono-modal symmetric peaks, and increment of molecular weight for BP was observed. These results also indicate that the well-defined BP was successfully synthesized. The number average molecular weights for the precursor and BP are $1.06 \times 10^{4}$, and $2.03 \times 10^{4}$, respectively. The DSC curves of PF and BP are shown in Figure 3. Glass transition temperature $T_{\mathrm{g}}$ of $\mathrm{PF}$ was $126{ }^{\circ} \mathrm{C}$. On the other hand, BP showed double $T_{\mathrm{g}}$ at 119 and $173{ }^{\circ} \mathrm{C}$, corresponding to $T_{\mathrm{g}}$ values of PF and PTAA blocks, respectively as observed in PTAA- $b$-PF- $b$-PTAA block copolymers without BT junctions [5], suggesting that BP shows a microphase-separated structure.

Figure 2

Figure 3 
The optical properties of synthesized polymers as well as blends [Blend $1 ; \mathrm{PF}+5 \mathrm{wt} \%$ of BP, Blend 2; PF + $10 \mathrm{wt} \%$ of BP, Blend 3; PF + model compound: 4,7-bis[4- $(N-4-$ butylphenyl- $N$-phenyl)aminophenyl]-2,1,3-benzothiadiazole (TAABT)] were investigated by UV-vis absorption and PL measurements in chloroform solution and in film state. The results of each sample are summarized in Table 2. Figures 4 (a) and (b) show UV-vis absorption spectra for the precursor and BP, respectively. As observed in PTAA- $b$-PF- $b$-PTAA [5], no significant difference was observed in the spectra in the solution and the film state. Both spectra exhibit absorption maximum around $390 \mathrm{~nm}$, and absorption tail due to the BT moiety in the region from 400 to $500 \mathrm{~nm}$. Figures 4 (c) and (d) show PL spectra for the precursor and BP. In the solution, emission from PF moiety is dominant, and a slight emission from incorporated BT unit is observed in 520-600 nm, while in the film state, the emission from BT moiety is clearly observed in both polymers. This is due to Förster type energy transfer from PF block to BT unit because of the overlap of absorption spectrum of BT unit and the emission spectrum of PF.

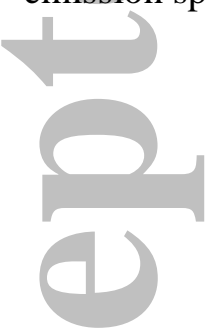

Table 2

Figure 4

Figures 5 (a), (b), and (c) show UV spectra for Blends 1-3. The content of TAABT was adjusted at $0.04 \mathrm{wt} \%$ corresponding to the same concentration of BT unit $(0.07 \mathrm{wt} \%)$ for 10\% Blend 2. As expected, all the blend samples exhibit the similar spectrum, where the negligible absorption for BT moiety is observed. Figures 5 (d), (e), and (f) show PL spectra for the same blends. For the solution samples, emission from PF component is predominately observed, and that from BT moiety is negligible due to diluted BT unit, while for the film samples, emission behaviors are different each other. Blend 1 and Blend 2 exhibit both emissions from PF component and BT unit, where the intensity ratio of PF block for Blend 1 
is larger than Blend 2 simply due to the difference of PF ratio. It is noteworthy that Blend 3 shows larger emission from BT unit compared with Blend 2, although both blends have the same concentration of BT unit. This is probably due to the difference of distribution of BT unit in the thin films. In the case of Blend 2, it is considered that BT moieties exist at the interface between the PF and PTAA domains in the phase separated structure due to the incorporation of BT unit at the junction. On the other hand, in the film of Blend 3, it is reasonable that TAABT is molecularly dispersed resulting in more efficient Förster type energy transfer from PF to TAABT.

Figure 5

\subsection{Device Performance}

The performance of PLEDs based on BP and PF blended with BP and TAABT with the device configuration of ITO/PEDOT:PSS/polymer/BCP/LiF/Al was evaluated. Figure 6 shows EL spectra for BP based device at various voltages. This device exhibited orange emission with CIE coordinate of $(0.52,0.47)$ at $15 \mathrm{~V}$. In the case of PL spectrum as shown in Figure $4 \mathrm{~d}$ ), the thin film showed slight emission in the blue region, while no blue emission was observed in EL spectrum. This result makes it possible to speculate that BT moiety in BP plays a role of a trap site as pointed out by Liu et al. $[12,21]$. It can be assumed that HOMO and LUMO energy levels of BT moiety seem similar to that of TAABT, -5.25 and $-3.06 \mathrm{eV}$, respectively [12], which lie between HOMO of PTAA $(-5.3 \mathrm{eV})$ [5] and LUMO of PF (-2.70 eV) [27]. Cyclic voltammogram of BP is presented in Figure S4. Indeed, HOMO and LUMO level of BP were estimated to be $-5.32 \mathrm{eV}$ and $-2.72 \mathrm{eV}$ determined from each onset point of oxidation and reduction waves in $\mathrm{CV}$, respectively. Therefore, if hole and electron transporting predominantly occurs in phase separated PTAA and PF domains, respectively, 
BT moiety at the interface effectively acts as the trap for both of hole and electron. As shown in Figure 6, voltage dependence for emission spectrum was negligible, indicating the color stability is achieved in this system.

Figure 6

Figure 7 represents current density-voltage (a), current efficiency-current density (b), and luminance-voltage (c) curves for the devices with BP, and Blends 1-3. PLED properties of synthesized polymers and blends are summarized in Table 3. The PLED performance of our system is low as compared with other white PLED [12]. However, we disclosed novel concepts for the design to improve PLED performance as follows. In the present case, the higher current efficiency and luminescence were observed in BP and Blend 2 based device. This is probably due to the high hole injection and/or transporting PTAA content leading to preferable balance of hole and electron. Judging from CIE coordinates listed in Table 3, and photographs of emission from the devices based on blends in Figure 8, characteristic emission behavior was observed in the BP doped devices. In the case of Blend 3, where low molecular model compound TAABT was utilized, orange emission was observed with CIE coordinate of $(0.58,0.42)$. On the other hand, Blend 2, where BT content was the same as in Blend 3, showed pale orange with the coordinate of $(0.44,0.38)$ at $7 \mathrm{~V}$. This emission appeared to be near-white to the naked eye as shown in Figure 8. Blend 1 based device exhibited the emission with the coordinate of $(0.34,0.41)$ approaching to ideal white emission due to the well-balanced blue and its complementary color orange emission. These results can be interpreted by the assumption that the segregation of PTAA part at the interface between PEDOT:PSS and occurred in the thin film Blend 1 and Blend 2 due to the strong interaction of hydrophilic TEO group with PEDOT:PSS. As a result, orange emitting BT moiety locates near the anode side in the blends. It is considered that this segregation makes simultaneous 
blue and orange emission possible. On the other hand, low molecular TAABT distributes homogeneously in PF matrix resulting in the orange emission.

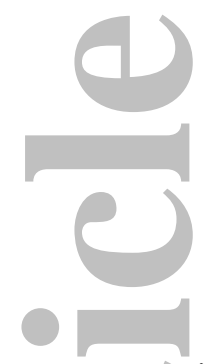

Figure 7

Table 3

Figure 8

Figure 9 shows EL spectra for Blend 2 based device at 7 and $10 \mathrm{~V}$. The CIE coordinates was $(0.44,0.38)$ and $(0.48,0.45)$ at 7 and $10 \mathrm{~V}$, respectively. Although both of blue and orange emissions are observed, the blue contribution from PF moiety was decreased with the increase of the applied voltage, which is similar to the results in the literature [12]. Inverse bias dependence, i.e., increase of blue contribution with the bias, has been also reported in the literatures [28-30]. This inverse contribution changes in dopant based white PLEDs, has been usually attributed to the very low doping concentration of the narrow energy gap dopants (not higher than $1 \mathrm{wt} \%$ ). The possibility of charge trapping on such dopants or energy transfer from a wide energy gap dopant will decrease when the low energy excited sites are saturated at the higher current density [28]. This bias dependence has been also explained by a field-dependent competition between hole trapping on narrow band gap dopants and undisturbed hole transport across the emissive layer [30]. In an active layer here, the concentration of BT moiety with narrow band gap is higher than in others ever reported. Therefore, the former explanation cannot be adopted to our system. It is generally considered that emission stability is achieved by extremely well-balanced electron and hole distribution in devices consisting of a two-layer stack [31], or by the strict control of recombination site [32]. In our systems, it can be considered that by changing the ratio hole and electron with the bias, the recombination site becomes closer to the anode leading to the increase of trap possibility at BT moiety, which segregates in the vicinity of the anode as mentioned above. 
Figure 9

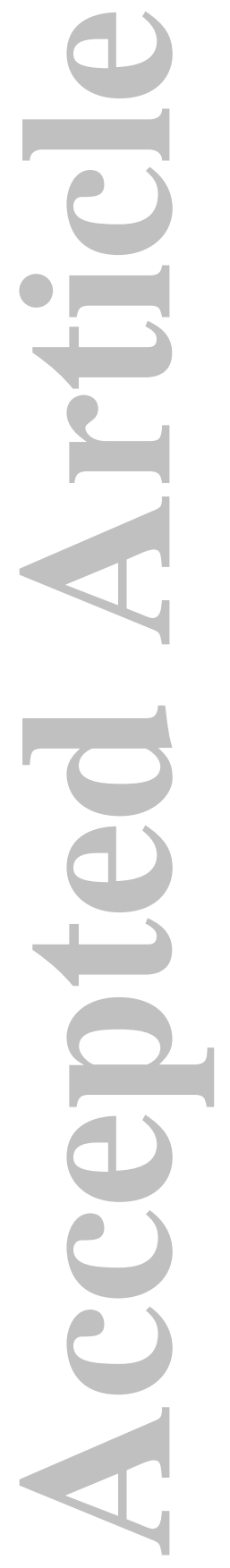

This article is protected by copyright. All rights reserved. 


\section{Conclusions}

Novel type of block copolymer consisting of hole transporting polytriarylamine (PTAA) and electron transporting polyfluorene (PF) chemically modified with benzothiadiazole (BT) moiety at the junction was designed. Resulting block copolymer afforded orange light emitting PLED. By the comparison of EL and PL spectra in film state, it is concluded that incorporated BT at the junction plays a role of an effective trap site. The characteristics of the block copolymer as a dopant were also evaluated. It is suggested that block copolymer preferentially distributes at the vicinity of anode via hydrophilic interaction of trioxyethylene side chains in PTAA block with PEDOT/PSS, while a low molecular dopant homogeneously distributes in the film. Molecular design presented here would afford new strategies for improved performance in PLEDs.

\section{Acknowledgement}

This research was partially supported by Grant-in-Aid for Scientific Research (KAKENHI, Grant number 24550209) from Japan Society for the Promotion of Science (JSPS). 


\section{References}

[1] J. H. Burroughes, D. D. C. Bradley, A. R. Brown, R. N. Marks, K. Mackay, R. H. Friend, P. L. Burns and A. B. Holmes, Nature 1990, 347, 539.

[2] L. Akcelrud, Prog. Polym. Sci. 2003, 18, 875.

[3] a) Y. Ohmori, M. Uchida, K. Muro and K. Yoshino, Jpn. J. Appl. Phys., Part 2 1991, 30 1941; b) M. Leclerk, J. Polym. Sci. Part A: Polym. Chem. 2001, 39, 2867.

[4] a) S. K. Lee, T. Ahn, N. S. Cho, J. Polym. Sci. Part A: Polym. Chem. 2007, 45, 1199;

b) M. Redecker, D. D. Bradley, M. Inbasekaran , W. W. Wu, E. P. Woo, Adv. Mater. 1999, 11, 241; c) C. Ego , A. Grimsdale , F. Uckert, G. Yu , G. Srdanov, K. Müllen, Adv. Mater. 2002, 14, 809; d) T. Miteva , A. Meisel, W. Knoll , H. G. Nothofer , U. Scherf , D. C. Müller, K. Meerholz, A. Yasuda, D. Neher, Adv. Mater. 2001, 13, 565; e) C. W. Wu, H. C. Lin, Macromolecules, 2006, 39, 7232.

[5] Y. Tan, Z. Gu, K. Tsuchiya, K. Ogino, Polymer 2012, 53, 1444.

[6] K. Tsuchiya, T. Shimomura, K. Ogino, Polymer 2009, 50, 95.

[7] Y. Tan, K. Tsuchiya, K. Ogino, Chem. Lett. 2012, 41, 257.

[8] S. T. Huang, D. J. Liaw, L. G. HsieH, C. C. Chang, M. K. Leung, K. L. Wang, W.T. Chen, K. R, Lee, J. Y. Lai, L. H. Chan, C.T. Chen, J. Polym. Sci. Part A: Polym. Chem. 2009, 47, 6231 .

[9] R. Guan, C. Li, W. Yang, W. Sun, H. Wu, L. Ying, Y. Cao, Dyes Pigm. 2008, 78, 165. [10] M. J. Park, J. Lee, I. H. Jung, J. H. Park, D. H. Hwang, H. K. Shim, Macromolecules 2008, 41, 9643 .

[11] C. Y. Chuang, P. I. Shih, C. H. Chien, F. I. Wu, C. F. Shu, Macromolecules, 2007, 40, 247.

[12] J. Liu, Q. Zhou, Y. Cheng, Y. Geng, L. Wang, D. Ma, X. Jing, F. Wang, Adv. Funct. Mater. 2006, 16, 957. 
[13] L. Chen, P. Li, H. Tong, Z. Xie, L. Wang, X. Jing, F. Wang, J. Polym. Sci. Part A: Polym. Chem. 2012, 50, 2854.

[14] H. J. Song, D. H. Kim, T. H. Lee, D. K. Moon, Eur.Polym. J. 2012, 48, 1485.

[15] L. Yu, J. Liu, S. Hu, R. He, W. Yang, H. Wu , J. Peng, R. Xia, D. D. C. Bradley, Adv.

Funct. Mater. 2013, 23, 4366.

[16] B. Liu, W. L. Yu, Y. H. Lai, W. Huang, Macromolecules 2000, 33, 8945.

[17] N. S. Cho, D. H. Hwang, J. I. Lee, B. J. Jung, H. K. Shim, Macromolecules 2002, 35, 1224.

[18] D. Cao, Q. Liu, W. Zeng, S. Han, J. Peng, S. Liu, Macromolecules, 2006, 39, 8347.

[19] X. Gong, W. L. Ma, J. C. Ostrowski, G. C. Bazan, D. Moses, A. J. Heeger, Adv. Mater. 2004, 16, 615 .

[20] J. Liu, L. Chen, S. Shao, Z. Xie, Y. Cheng, Y. Geng, L. Wang, X. Jing, F. Wang, J. Mater. Chem. 2008, 18, 319.

[21] J. Liu, X. Guo, L. Bu, Z. Xie, Y. Cheng, Y. Geng, L. Wang, X. Jing, F. Wang, Adv. Funct. Mater. 2007, 17, 1917.

[22] T. Virgili, D. G. Lidzey, D. D. C. Bradley, Adv. Mater. 2000, 12, 58.

[23] Q. Xu, H. M. Duong, F. Wudl, Y. Yang, Appl. Phys. Lett. 2004, 85, 3357.

[24] H. A. Al Attar, A. P. Monkman, M. Tavasli, S. Bettington, M. R. Bryce, Appl. Phys. Lett. 2005, 86, 121101.

[25] M. Jahanfar, K. Suwa, K. Tsuchiya, K. Ogino, Open J. Org. Polym. Mater. 2013, 3, 46.

[26] K. Tsuchiya, T. Sakakura, K. Ogino, Macromolecules 2011, 44, 5200.

[27] D. Neher, Macromol. Rapid Commun. 2001, 22, 1365.

[28] J. Huang, G. Li, E. Wu, Q. Xu, Y. Yang, Adv. Mater. 2006, 18, 114.

[29] X. Gong, S. Wang, D. Moses, G. C. Bazan, A. J. Heeger, Adv. Mater. 2005, 17, 2053. 
[30] J. Zou, H. Wu, C. S. Lam, C. Wang, J. Zhu, C. Zhong, S. Hu, C. L. Ho, G. J. Zhou, H.

Wu, W. C. H. Choy, J. Peng, Y. Cao, W. Y. Wong, Adv. Mater. 2011, 23, 2976

[31] A. Köhnen, M. Irion, M. C. Gather, N. Rehmann, P. Zacharias, K. Meerholz, J. Mater. Chem., 2010, 20, 3301.

[32] N. Aizawa, Y.-J. Pu, M. Watanabe, T. Chiba, K. Ideta, N. Toyota, M. Igarashi, Y. Suzuri, H. Sasabe, J. Kido, Nat. Commun. 2014, 5, 5756.

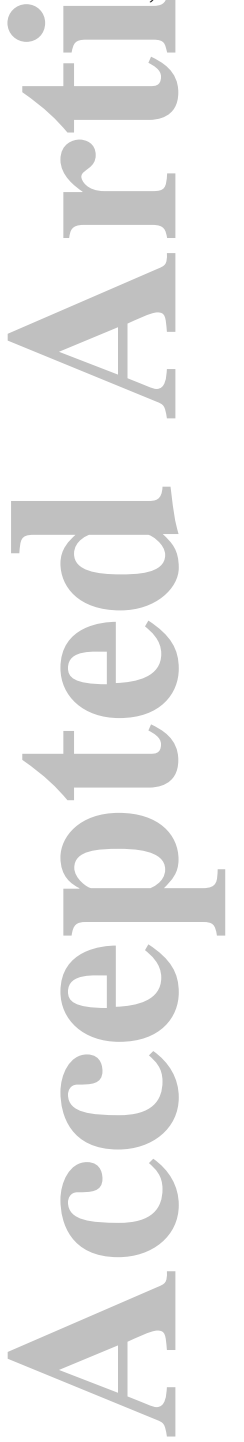




\section{Figure caption}

Scheme 1 Chemical structures of (a) BP (PTAA- $b$-PF- $b$-PTAA block copolymer functionalized with BT moiety at the junction point of two blocks), (b) PF, and (c) BT.

Figure $1 \quad{ }^{1}$ H-NMR spectra of (a) BP precursor, (b) BP.

Figure 2 GPC trace of (a) polyfluorene, (b) BP precursor, and BP.

Figure 3 DSC curves of PF and BP.

Figure 4 Optical properties of BP precursor and BP: (a) UV-vis absorption spectra of BP precursor; (b) UV-vis absorption spectra of BP; (c) PL spectra of BP precursor; (d) PL spectra of BP.

Figure 5 Optical properties of Blends 1-3 [Blend 1; PF $+5 \mathrm{wt} \%$ of BP, Blend 2; PF +10 wt\% of BP, Blend 3; PF + model compound: 4,7-bis[4-( $N$-4-butylphenyl- $N$ phenyl)aminophenyl]-2,1,3-benzothiadiazole (TAABT)]: (a), (b), (c) UV-vis spectra of blend 1, 2, 3; (d), (e), (f) PL spectra of Blends 1-3.

Figure 6 Electroluminescence spectra of PLED device based on BP at 15, 20, $32 \mathrm{~V}$.

Figure 7 Device characteristics of BP and Blends 1-3, (a) current density - voltage characteristics; (b) current efficiency - voltage characteristics; (c) luminance voltage characteristics.

Figure 8 Photographs of PLED devices of Blends 1-3.

Figure 9 Electroluminescence spectra of PLED device based on Blend 2 at 7, $10 \mathrm{~V}$. 


\section{University Library}

\section{- M I I N E R VA \\ A gateway to Melbourne's research publications}

Minerva Access is the Institutional Repository of The University of Melbourne

Author/s:

Kim, K;Inagaki, Y;Kanehashi, S;Ogino, K

Title:

Incorporation of benzothiadiazole moiety at junction of polyfluorene-polytriarylamime block copolymer for effective color tuning in organic light emitting diode

Date:

2017-11-05

\section{Citation:}

Kim, K., Inagaki, Y., Kanehashi, S. \& Ogino, K. (2017). Incorporation of benzothiadiazole moiety at junction of polyfluorene-polytriarylamime block copolymer for effective color tuning in organic light emitting diode. Journal of Applied Polymer Science, 134 (41), pp.45393-45393. https://doi.org/10.1002/app.45393.

Persistent Link:

http://hdl.handle.net/11343/293090 